\title{
Influencia de los vertimientos mineros sobre las características fisicoquímicas del agua de cuatro fuentes hídricas de la zona norte del Chocó Biogeográfico
}

\section{Influence of miners dumping on water physiochemical characteristics four rivers of the north Chocó Biogeográfico}

\section{Fairy María Medina Mosquera*}

\section{Resumen}

A partir de la identificación de cuatro fuentes hídricas, receptoras de vertimientos mineros presentes en los municipios de Condoto, Tadó y Quibdó, se analizó la influencia de los efluentes procedentes de la minería auroplatinifera sobre las características físico-químicas del agua, en tres puntos diferentes distribuidos antes, durante y después de los vertimientos, mediante la aplicación de mediciones in situ y análisis de muestras de aguas en laboratorio. Se determinó que los vertimientos mineros ejercen una influencia estadísticamente significativa especialmente sobre las variables sólidos totales y sólidos suspendidos, aunque para las variables turbiedad y $D Q O$, se registraron diferencias estadísticas significativas entre las estaciones antes y durante, y entre antes y después de los vertimientos mineros. Por otro lado, aunque no se observó el mismo comportamiento para variables como mercurio y grasas y aceites; en algunos puntos las concentraciones estuvieron por fuera de los valores establecidos por la normatividad nacional e internacional en aguas para consumo humano y uso doméstico y en aguas para conservación de flora y fauna acuática, a diferencia del $\mathrm{pH}$, quien registró valores por dentro del rango establecido para ambos usos. En cuanto a la BBO5, esta fue constante y relativamente muy baja para todos los puntos analizados en las cuatro fuentes hidricas.

Palabras clave: Contaminación hídrica, Distrito Minero del San Juan, Efectos de la minería aluvial.

\begin{abstract}
From the identification of four water sources, receiving mining discharges present in the municipalities of Condoto, Tadó and Quibdó, the influence of effluents from the auroplatinifera mining on the physico-chemical characteristics of water was analyzed at three different points distributed before, during and after dumping. Above, by applying in situ and analyzing water samples in laboratory measurements. It was determined that the miners vertimientos exert a statistically significant influence especially on solid variables totals and suspended solids, although for turbidity and DQOvariables, statistically significant between the stations before and during differences were recorded, and between before and after the miners vertimientos. On the other hand, although not the same behavior for variables like mercury and fats and oils, at some points the concentrations were outside the values established by national and international standards in water for human consumption and domestic use and water for observed conservation of aquatic flora and
\end{abstract}

* Ingeniera Ambiental. Investigadora del Instituto de Investigaciones Ambientales del Pacífico (IIAP), Quibdó, Colombia.

e-mail: fmedina@iiap.org.co

Recibido: 5 de diciembre de 2012

Aceptado: 20 de marzo de 2013 


\section{Bioetnia Volumen 10, 2013}

fauna, unlike the $p H$, who recorded values within the range established for both uses. Regarding DBO5, this was constant and relatively very low for all four points analyzed in water sources.

Keywords: Effects of alluvial mining, Mining district of San Juan, Water pollution.

\section{Introducción}

La minería en pequeña escala, tradicional y de hecho, ha sido reconocida por distintos instrumentos internacionales como un tipo de producción que tiene profundas raíces históricas y sociales y que está asentado en la cultura y la economía de América Latina (Defensoría del Pueblo 2010). Esta acción extractiva especialmente de oro y platino en el Pacífico colombiano es una de las principales actividades económica de sustentabilidad de la población afrodescendiente (IDRC-IIAP 2004). Para el caso del departamento del Chocó, ha sido desarrollada de manera intensiva especialmente en los municipios presentes en la cuenca del río San Juan y en la parte Alta y Media del río Atrato, resaltando entre estos los municipio de Condoto, Istmina, Tadó, Unión Panamericana, Medio San Juan, Quibdó, Lloró, Bagadó, entre otros.

Esta actividad se ha desarrollado mediante la aplicación de sistemas de explotación artesanal, semi-mecanizado y mecanizado a lo largo de la costa pacífica colombiana. Debido a los altos precios del oro, en las últimas décadas la minería aurífera en suelos aluviales se ha expandido más rápidamente que en cualquier época histórica, generando enormes costos para la salud humana y para el medio ambiente en general (Brack et al. 2011). Su intensificación ha traído nefastas consecuencias para los ecosistemas, con una agravio especial para el recurso hídrico, el cual ha convertido a la minería de hecho en el problema más sensible desde el punto de vista del impacto ambiental que tiene hoy en día el país, los cuales se deben principalmente a dos factores, entre ellos, la utilización de toneladas de productos químicos que terminan en los ríos circunvecinos y en las aguas freáticas, generando problemas de salubridad pública irreversibles, con consecuencias a corto y largo plazo (Defensoría del Pueblo 2010). De igual forma, está ocasionando la sedimentación de los cuerpos de agua, alteración de los cauces, erosión de las orillas y disminución del recurso ictiológico. Su expansión ha alcanzado innumerables fuentes hídricas de gran importancia sociocultural en el Distrito Minero del San Juan, entre las cuales se destacan los ríos Condoto, Tamaná, Iró, Taparal, San Pablo, Tadocito, Opogodó, las quebrada Pureto y Tapacundó, y sus afluentes, entre otras, que se constituyen en las principales fuentes hídricas de la región, caracterizadas por su importante diversidad de especies ícticas y forestales, y con capacidad de albergar cientos de especies faunísticas nativas de la zona.

Dada la importancia económica y cultural que representa la actividad minera para la región del San Juan y para la parte alta y media de la cuenca del río Atrato, ante los vacíos de información que se presentan frente a las afectaciones que se podrían estar generando por el desarrollo de la minería de oro y platino sobre el recurso hídrico, se sintió la necesidad de investigar e indagar sobre la influencia que generan los efluentes mineros sobre las características fisicoquímicas del agua de las fuentes hídricas intervenidas en la zona norte del Chocó Biogeográfico.

\section{Metodología}

Área de muestreo. De las cuatro fuentes hídricas analizadas, tres se encuentran ubicadas en el Distrito Minero del San Juan y corresponden a los ríos Tadocito y Pureto en el municipio de Tadó y a la quebrada Tapacundó en el municipio de Condoto. La otra fuente hídrica corresponde a la quebrada Esperanza, la cual se encuentra ubicada en el corregimiento de Guayabal, municipio de Quibdó (Figura 1). La quebrada Tapacundó, corresponde a una fuente hídrica de tercer orden, receptora de vertimientos mineros procedentes de la mina Nicole. Nace en la Cuchilla de Máncamo y tributa sus aguas al río Opogodó. Recorre las comunidades de Jigualito, El Guamo, El Tigre y Opogodó. Su caudal es aproximadamente de $4.576 \mathrm{~m}^{3} \mathrm{seg}^{-1}$. El substrato es pedregoso, con cantos redondeados de baja esfericidad entre $1 \mathrm{~cm}$ y $30 \mathrm{~cm}$ (promedio $5 \mathrm{~cm}$ ). El río Tadocito, corresponde a una fuente hídrica de segundo orden, receptora de vertimientos mineros procedentes de la mina La Platina. Tributa sus aguas al río San Juan y recorre las comunidades del Tapón, Tadocito, La Esperanza y Bapura. Su caudal es aproximadamente de $3.11 \mathrm{~m}^{3} \mathrm{seg}^{-1}$ y presenta un substrato pedregoso. El río Pureto, receptor de los vertimientos de la mina La Esperanza, recorre las comunidades de Carmelo y Playa de Oro en el municipio de Tadó, y su caudal es aproximadamente de $1.04 \mathrm{~m}^{3} \mathrm{seg}^{-1}$. La quebrada Esperanza desemboca en el río Hugón, a la altura de la comunidad de Guayabal, esta presentó un ancho de un metro y una profundidad promedio de $30 \mathrm{~cm}$, se encuentra represada en su parte alta, condición que impidió apreciar y calcular la velocidad de la corriente. En el último punto de muestreo presentó un caudal de $0.5636 \mathrm{~m}^{3} \mathrm{seg}^{-1}$, con un substrato con alto contenido de arenas y arcillas. A diferencia de las estaciones de muestreos ubicadas después de los vertimientos mineros, antes de ellos, el agua de estas fuentes hídricas en términos generales es cristalina, y sin presencia de olor o sabor, además con bajos niveles de turbiedad y presencia 
Vertimientos mineros y fuentes hídricas. FM Medina
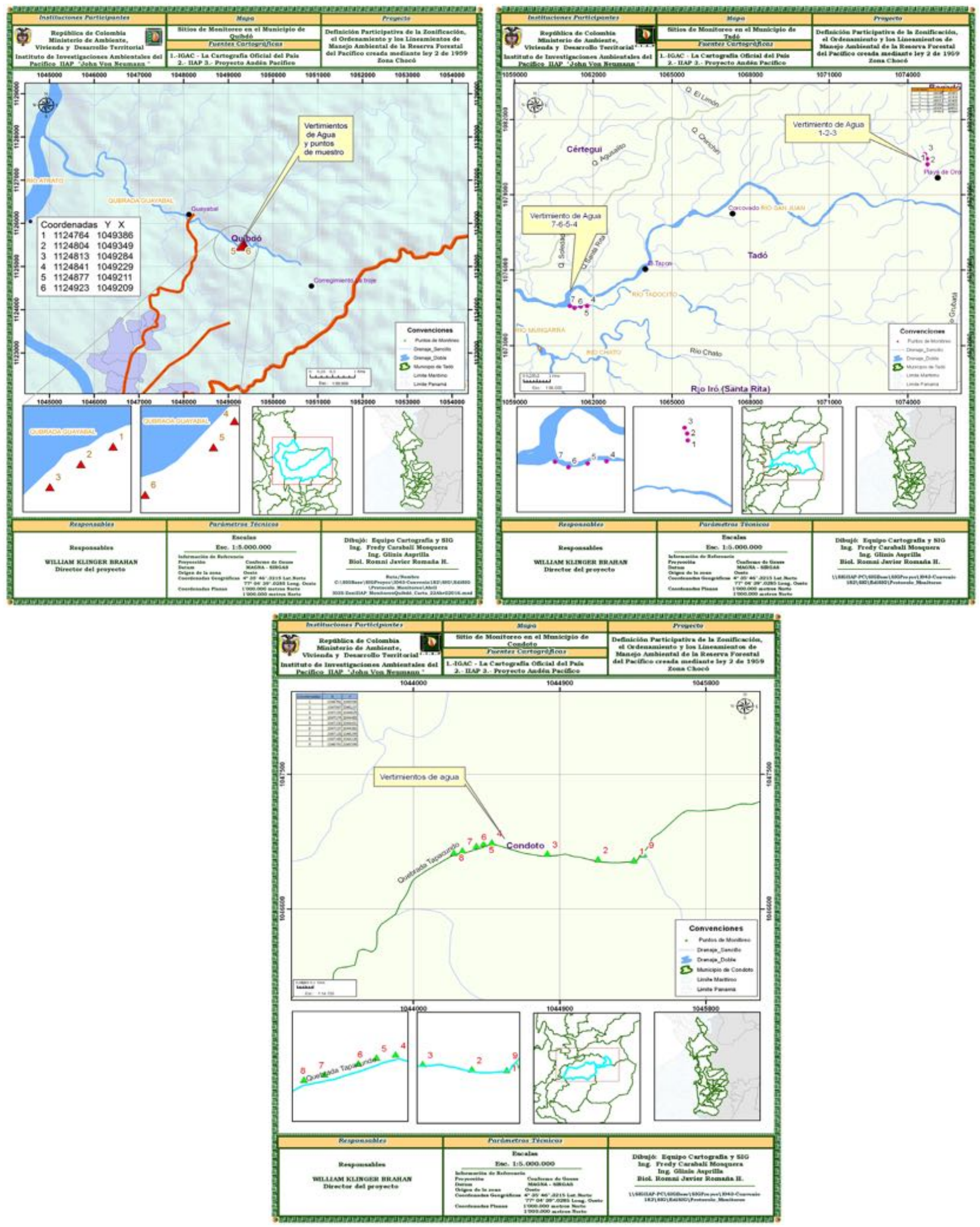

Figura 1. Ubicación geográfica del área de estudio: quebrada Tapacundó, municipio de Condoto; ríos Pureto y Tadocito, en el municipio de Tadó; quebrada La Esperanza, municipio de Quibdó. 


\section{Bioetnia Volumen 10, 2013}

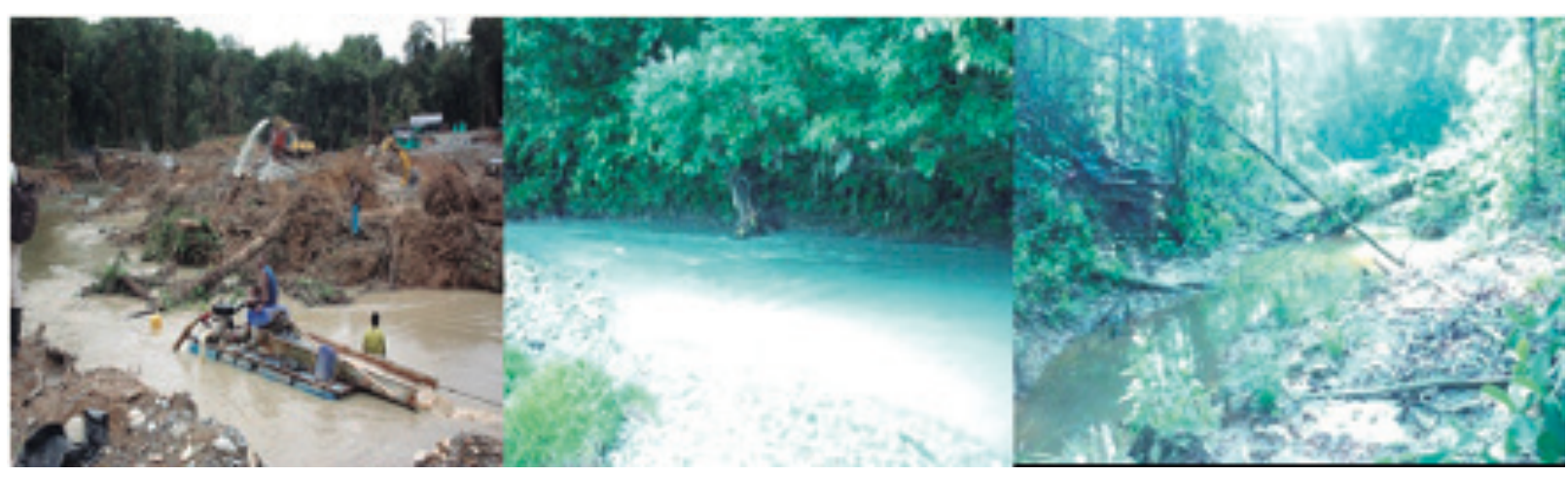

Figura 2. Características generales de las fuentes hídricas analizadas en los municipios de Condoto, Tadó y Quibdó.

de sedimentos (Figura 2).

Métodos. Para determinar la influencia de la actividad minera sobre las características fisicoquímicas del agua se definieron tres estaciones de muestreos en cada uno de las cuatro fuentes hídricas analizadas. Estas estaciones fueron ubicadas antes, durante y después de los vertimientos mineros procedentes de las minas Nicole, La Platina, La Esperanza y de una unidad productiva familiar de minería semi-mecanizada (minería de chorro), las cuales fueron previamente seleccionadas por su facilidad de acceso y ubicación con respecto al cauce de estas fuentes hídricas. En cada sitio se realizaron mediciones in situ y toma de muestras de aguas puntuales, para el análisis de parámetros físicos como sólidos suspendidos (SS), sólidos totales (ST) y turbiedad y químicos como oxígeno disuelto, $\mathrm{pH}$, grasas y aceites, mercurio total (Hg-T), demanda química de oxígeno (DQO) y demanda bioquímica de oxígeno $\left(\mathrm{DBO}_{5}\right)$. $\mathrm{El}$ análisis de estos parámetros se debe a la relación que existe entre ellos, la cual puede ser directa o inversamente proporcional según las concentraciones de la carga contaminante procedente del lavado de la grava auroplatinífera.

Una vez obtenidos los resultados de cada una de las variables físico-químicas analizadas, estos fueron comparados con los estándares de calidad de agua establecidos para la destinación del recurso hídrico para consumo humano y uso doméstico y para conservación de flora y fauna acuática establecidos por el Decreto 1594 del 1984 y por algunas normativas internacionales, entre ellas: las Guías de Calidad para Agua de Bebida del Canadá y las Guías de Calidad para Aguas de Consumo Humano de la Organización Mundial de la Salud (OMS) Además se aplicó un análisis de varianza de un factor para cada una de las variables fisicoquímicas estudiadas. De este análisis se tomaron los resultados obtenidos en la Prueba de Kruskal-Wallism, donde se compararon las medianas en lugar de las medias, porqie los datos no tenían una distribución lineal normal. Esta prueba evaluó la hipótesis de que las medianas de cada una de las variables estudiadas dentro de cada uno de los 3 niveles de estaciones de muestreos son iguales. En este caso, primero se combinaron los datos de todos los niveles y se ordenaron de menor a mayor y luego se calculó el rango promedio para los datos de cada nivel. Valores de p iguales o menores a 0.5 , indican que existe una diferencia estadísticamente significativa entre las medianas, y valores iguales o mayores de 0.05 indican lo contrario; para ambos casos con un nivel de $95 \%$ de confianza.

\section{Resultados y discusión}

Variación de las características fisicoquímicas de las fuentes hídricas estudiadas por estaciones de muestreos. Los resultados de las variables fisicoquímicas analizadas en las quebradas Tapacundó y La Esperanza y en los ríos Pureto y Tadocito, mostraron que los parámetros con mayores reportes fueron los sólidos suspendidos, sólidos totales, turbiedad y la DQO (Tabla 1). En cuanto a los resultados del análisis de varianza ANOVA, las variables turbiedad, $\mathrm{pH}$, grasas y aceites, DBO5, DQO, y mercurio, registraron un valor $\mathrm{p}$ a partir de la prueba de Kruskal-Wallis iguales o mayores a $0,05 \%$, y para los sólidos suspendidos y sólidos totales, por debajo de $0,05 \%$ (Tabla 2).

Teniendo en cuenta lo anterior, las concentraciones de turbiedad antes de los vertimientos presentaron un valor de 0,0 UNT para los ríos Tadocito y Pureto y un valor de 35 y 166 UNT para las quebradas La Esperanza y Tapacundo. Para las estaciones durante y después de los vertimientos, estas concentraciones oscilaron entre 72 y 1100 UNT, reportándose los niveles más bajos en el río Pureto y la quebrada Tapacundó y los más altos para el río Tadocito y la quebrada La Esperanza (Tabla 1). Las diferencias de turbiedad entre las estaciones de muestreos ubicadas antes de los vertimientos, especialmente la que corresponde a la quebrada Tapacundó con respecto a las otras tres fuentes hídricas, podría estar relacionada con la presencia de lluvias previo a las mediciones realizadas, porque en el momento del muestreo no se identificó ningún 
Tabla 1

Resultados físico-químicos obtenidos en la quebrada Tapacundó y en los ríos Pureto y Tadocito

\begin{tabular}{|c|c|c|c|c|c|c|c|c|c|}
\hline \multicolumn{2}{|c|}{ Puntos de muestreos } & \multicolumn{8}{|c|}{ Parámetros fisicoquímicos } \\
\hline Fuentes hídricas & Puntos de muestreo & Tub & $\begin{array}{c}\text { SS } \\
\text { (mg/l) }\end{array}$ & $\begin{array}{c}\text { ST } \\
(\mathrm{mg} / \mathrm{l})\end{array}$ & $\mathrm{pH}$ & $\begin{array}{l}\text { GyA } \\
\text { (mg/l) }\end{array}$ & $\begin{array}{c}\text { DBO5 } \\
\text { (mg/l) }\end{array}$ & $\begin{array}{l}\text { DQO } \\
(\mathrm{mg} / \mathrm{l})\end{array}$ & $\begin{array}{l}\text { MER } \\
(\mathrm{mg} / \mathrm{l})\end{array}$ \\
\hline \multirow[t]{3}{*}{ Tapacundó } & Antes del vertimiento & 166 & 42 & 120 & 7,2 & 76,25 & 2,00 & 21,8 & 0,00 \\
\hline & Durante el vertimiento & 276 & 430 & 516 & 7,6 & 71,75 & 2,00 & 36,4 & 0,00 \\
\hline & Después del vertimiento & 320 & 700 & 860 & 7,1 & 71,25 & 2,00 & 72,7 & 0,00 \\
\hline \multirow[t]{3}{*}{ Pureto } & Antes del vertimiento & 0 & 7 & 65 & 5,7 & 5,00 & 2,00 & 12,0 & 0,001 \\
\hline & Durante el vertimiento & 81 & 798 & 858 & 6,7 & 5,00 & 2,00 & 12,0 & 0,001 \\
\hline & Después del vertimiento & 72 & 356 & 410 & 6,6 & 5,00 & 2,00 & 12,0 & 0,002 \\
\hline \multirow[t]{3}{*}{ Tadocito } & Antes del vertimiento & 0,0 & 10,3 & 12 & 5,9 & 0,00 & 2,00 & 58,2 & 0,00 \\
\hline & Durante el vertimiento & 1100 & 2827 & 3225 & 8,7 & 5,00 & 2,00 & 280,0 & 0,003 \\
\hline & Después del vertimiento & 874 & 1063 & 1234 & 8,7 & 5,00 & 2,00 & 96,5 & 0,001 \\
\hline \multirow[t]{3}{*}{ La Esperanza } & Antes del vertimiento & 35 & 12 & 53 & 6,3 & 5,00 & 2,58 & 12,4 & 0,001 \\
\hline & Durante el vertimiento & 1100 & 3735 & 4059 & 8,7 & 5,00 & 2,00 & 27,2 & 0,002 \\
\hline & Después del vertimiento & 1100 & 8799 & 10230 & 8,7 & 5,00 & 2,00 & 323,0 & 0,001 \\
\hline
\end{tabular}

Tabla 2

Resultados obtenidos en la prueba de Kruskal-Wallism

\begin{tabular}{|c|c|c|c|c|c|c|c|}
\hline \multirow[t]{2}{*}{ Comparación } & \multirow[t]{2}{*}{ Turbiedad } & \multirow[t]{2}{*}{$\mathrm{pH}$} & \multirow[t]{2}{*}{ Grasas y aceites } & \multirow[t]{2}{*}{ DQO } & \multirow[t]{2}{*}{ Mercurio } & \multicolumn{2}{|c|}{ Sólidos } \\
\hline & & & & & & suspendidos & totales \\
\hline $1 / 2$ & 0,0103 & 0,5542 & 0,7004 & 0,0162 & 0,2191 & 0,0000 & 0,0001 \\
\hline $1 / 3$ & 0,0147 & 0,4409 & 0,6925 & 0,0135 & 0,5836 & 0,0000 & 0,0000 \\
\hline $2 / 3$ & 0,8474 & 0,8521 & 0,9904 & 0,9206 & 0,4715 & 0,1585 & 0,1372 \\
\hline
\end{tabular}

tipo o foco de contaminación directa que pudiera alterar las condiciones naturales de este punto de la quebrada. Estadísticamente estas concentraciones de turbiedad, no presentaron diferencias significativas entre las medianas de las tres estaciones de muestreos con un nivel de confianza del $95 \%$, porque el valor $\mathrm{p}$ fue de 0,0551997 ; sin embargo, dadas las variaciones de las concentraciones de turbiedad que se presentaron entre las estaciones antes y durante los vertimientos ( $p$ de 0,0103 ) y entre antes y después de los vertimientos ( $\mathrm{p}$ de 0,0147 ), se estableció que entre las medianas de estas estaciones si se presentó estadísticamente una diferencia significativa (Tabla 2).

Por otro lado, al relacionar las concentraciones de turbiedad con los estándares de calidad establecidos principalmente en aguas para consumo humano y uso doméstico que para su potabilización requiere solo desinfección, se observó que los resultados obtenidos en todas las estaciones de muestreos de las fuentes hídricas analizadas, excepto los registrados en los puntos antes de los vertimientos de los ríos Tadocito y Pureto, se encontraban por arriba de lo que recomienda la normatividad nacional (10 NTU); de igual manera los establecidos por otras normas entre ellas, La Agencia de Protección Ambiental de Estados Unidos (EPA), quien indica que las aguas para consumo humano deben tener preferiblemente una UNT y en ningún caso más de cinco UNT. En el caso de Las Guías de Calidad para Agua de Bebida del Canadá y las Guías de Calidad en Aguas de Consumo Humano de la Organización Mundial de la Salud (OMS), se recomiendan como valor guía 5 UNT.

A pesar de que no se conocen los efectos directos de esta variable sobre la salud, esta afecta la calidad estética del agua, lo que muchas veces ocasiona el rechazo de los consumidores (Barrenechea 2013). La turbiedad es un factor importante en la producción de oxígeno en cuerpos de aguas superficiales, porque los altos valores de turbiedad limitan la penetración de los rayos del sol y con ello la fotosíntesis, por consiguiente, se considera que los efluentes mineros presentan cargas contaminantes como sedimentos, que alteran los niveles naturales de turbiedad del agua de las fuentes hídricas receptoras. 


\section{Bioetnia Volumen 10, 2013}

El pH por su parte osciló entre 5,7 y 7,2 unidades antes de los vertimientos reportándose el valor más bajo en el río Pureto y el más alto en la quebrada Tapacundó y osciló entre 6,6 y 8,7 unidades durante y después de los vertimientos, registrándose el valor más bajo en el río Pureto y el más alto en la quebrada La Esperanza y el río Tadocito (Tabla 1). Según el análisis estadístico, estas variaciones de $\mathrm{pH}$, no tuvieron una diferencia significativa entre las medianas de cada una de las estaciones de muestreos, ya que generaron una p de 0,0764856 , con un nivel de $95 \%$ de confianza (Tabla 2). Por otro lado, al comparar las unidades de $\mathrm{pH}$ obtenidas en cada una de las estaciones de las cuatro fuentes hídricas analizadas, con los estándares de calidad establecidos por la normatividad nacional, en aguas para conservación de flora y fauna acuática, y en aguas para consumo humano y uso doméstico, se observa que a pesar de los resultados del análisis estadístico, se registraron valores que aumentaron notablemente entre las estaciones de muestreos ubicadas antes de los vertimientos con aquellos ubicados durante y después de los mismos, especialmente en el río Tadocito y en la quebrada La Esperanza. Aun así, es importante anotar que en ninguno de los casos esta variable registró valores por fuera del rango establecido para tales usos, considerando estos valores normales en el comportamiento de esta variable para cuerpos de aguas superficiales. Por lo general las aguas naturales (no contaminadas) exhiben un $\mathrm{pH}$ en el rango de 5 a 9, rango que deberían presentar tanto aguas crudas como aguas tratadas, porque permite controlar los efectos en el comportamiento de otros constituyentes del agua (Barrenechea 2013), deduciendo que los vertimientos mineros no influyen notablemente en el pH del agua de los cuerpos receptores de aguas superficiales.

Las grasas y aceites, presentaron valores menores de 5 $\mathrm{mgl}^{-1}$ para las tres estaciones de la quebrada La Esperanza y los ríos Pureto y Tadocito, los cuales difirieron notablemente con los registrados en la quebrada Tapacundó oscilando estos entre 71,25 y 76, 25 $\mathrm{mgl}^{-1}$ (Tabla 1). Lo anterior contribuyó a que se generara un valor p de 0,865892 , lo que indica que no hubo estadísticamente una diferencia significativa entre las medianas por estaciones de muestreo con un nivel de confianza del 95\% (Tabla 2). No obstante, estas concentraciones de grasas y aceites superan notablemente los estándares de calidad establecidos por el Decreto 1594 de 1984, en aguas para conservación de la flora y fauna acuática, porque estas no deben superar $0.01 \mathrm{mgl}^{-1}$ (Tabla 3 ). En este sentido, la Fundación Mapfre (2005), manifiesta que las grasas y aceites presentes en las fuentes hídricas, además de producir un impacto estético, reducen la reoxigenación del agua, disminuyendo el oxígeno disuelto y absorbiendo la radiación solar, afectando la actividad fotosintética y en consecuencia, la producción interna de oxígeno disuelto.

De igual forma, sucedió en aguas para consumo hu- mano y uso doméstico, por lo que, deben estar totalmente ausentes, según el Decreto 1594 de 1984, de lo contrario, se haría necesario la potabilización mediante tratamiento convencional en caso de requerirse, proceso que se podría ver afectado, según la Fundación Mapfre (2005), por la presencia de este tipo de contaminante, pues encarecen los tratamientos de depuración y algunos aceites sobre todo los minerales, pueden ser muy tóxicos. En consecuencia, los vertimientos mineros sí podrían afectar las presencian de esta variable en los cuerpos de aguas superficiales, porque al alcanzar concentraciones de grasas y aceites hasta de $69.783 \mathrm{~kg} /$ año (Vargas 2012), al diluirse modificaría las condiciones naturales del agua.

La $\mathrm{DBO}_{5}$, a diferencia de los otros parámetros, fue constante en las tres estaciones de muestreos de las cuatro fuentes hídricas analizadas. Esta variable registró valores menores de 2 mg-1 (Tabla 1), arrojando un valor P de 0,367879 , lo que indica que, estadísticamente no hubo una diferencia significativa de las medianas entre estaciones de muestreo con un nivel de 95\% de confianza (Tabla 2). Lo anterior podría evidenciar que los efluentes mineros tienen poca presencia de materia orgánica, posiblemente por la remoción de la capa orgánica que se realiza previo al lavado de la grava auroplatinífera, proceso con el que se reduce en un alto porcentaje la presencia de este tipo de contaminante en los cuerpos de agua superficiales por causa de la minería mecanizada con retroexcavadora especialmente.

Con respecto a la DQO, en las estaciones de muestreos ubicadas Antes de los vertimientos mineros se registraron concentraciones que oscilaron entre 12 y 21,8 mgl-1, presentando la concentración más baja en la quebrada Pureto y la más alta el río Tadocito. En el caso de las estaciones durante y después de los vertimientos, está osciló entre 12 y 323 mgl-1. Los valores más bajos se reportaron en la quebrada Pureto y el más alto en la quebrada La Esperanza (Tabla 2). Gracias a estas variaciones de DQO, se obtuvo un valor $\mathrm{p}$ de 0,384553 , resultado que indica que en términos generales no hubo una diferencia estadísticamente significativa entre las medianas de las estaciones de muestreos, sin embargo, por las variaciones de DQO presentadas entre las estaciones antes y durante los vertimientos ( $p$ de 0,0162 ) y entre antes y después de los vertimientos ( $\mathrm{p}$ de 0,0135 ), se evidencia que entre las medianas de estos puntos de muestreos sí hubo diferencia estadísticamente significativas (Tabla 2). Y al relacionar las concentraciones de DQO, con las obtenidas en la $\mathrm{DBO}_{5}$, se podría determinar que la carga contamínate que presentan los efluentes mineros son más de tipo inorgánico que orgánico, pues las concentraciones de DQO excedieron hasta 6 veces más el valor de DBO5 para el caso del río Pureto y más de 10 para el río Tadocito y la quebrada Tapacundó. Por su parte BioyGeo (2012) y el Instituto Catalán (2004), manifiestan que fuentes hídri- 
cas con concentraciones de DQO mayores de 40 mgl-1, y hasta por arriba de $80 \mathrm{mgl}-1$, indican aguas fuertemente contaminadas con materia inorgánica, lo que quiere decir que tres de las cuatro fuentes hídricas estudiadas, podrían estar dentro de esta categoría. Lo anterior evidencia que las fuentes hídricas con presencia de vertimientos mineros registran concentraciones de DQO diferentes a las que registran aquellas sin ningún tipo de foco de contaminación.

Elmercurio presentó concentraciones que oscilaron entre 0,0 y 0,001 mgl-1 para las estaciones de muestreo antes de los vertimientos y entre 0,0 y 0,003 para las estaciones durante y después de los vertimientos. Para el caso de la quebrada Tapacundó no hubo registro de mercurio en ninguna de las estaciones. Por otra parte, se encontraron altas concentraciones de mercurio en los ríos Tadocito y Pureto sobre todo después de los vertimientos mineros (Tabla 1). Estas variaciones de mercurio generaron un valor $\mathrm{p}$ de 0,395561 , lo que indica que no hubo una diferencia estadísticamente significativa entre las medianas con un nivel del 95\% de confianza (Tabla 2). Por otro lado, al comparar estos valores con las concentraciones establecidas por la normatividad nacional encontramos que aunque estos valores no superaron los criterios de calidad establecidos en aguas para conservación de la flora y fauna acuática $(0,01$ mgl-1), sí superaron los valores establecidos para consumo humano (0,002 mgl-1), lo que podría generar un riesgo de contaminación mercurial principalmente para las personas de las comunidades de la zona (Tablas 3 y 4).

Se considera que las bajas concentraciones de mercurio encontradas en las estaciones de muestreo de las cuatro fuentes hídricas analizadas, no es un indicador de la utilización de bajas cantidades de mercurio en la actividad minera o
Tabla 3

Criterios de calidad del agua para

conservación de flora y fauna acuática, según el Decreto 1594 de 1984

Parámetro

Decreto 1594 de 1984

\begin{tabular}{lc} 
Películas de grasas y & \\
aceites $(\mathrm{mg} / \mathrm{l})$ & 0,01 \\
$\mathrm{Ph}$ & $4,5-9,0$ \\
Oxígeno disuelto $(\mathrm{mg} / \mathrm{l})$ & 4.0 \\
Mercurio $(\mathrm{mg} / \mathrm{l})$ & 0,01 \\
\hline
\end{tabular}

de poca contaminación mercurial de las fuentes hídricas, porque según Sánchez y Cañón (2010), en los entables mineros, es común el uso del mercurio en el tramo inicial del canalón con el fin de ayudar a retener las partículas de oro en forma de amalgama, proceso realizado en circuitos abiertos en detrimento de la salud de los trabajadores y de la calidad del medio ambiente. Por su parte CODECHOCO (2010), indica que a las corrientes hídricas llegan $30 \mathrm{~kg} / \mathrm{año}$ por entable en el departamento del Chocó. En otros estudios se ha establecido que por cada $1 \mathrm{~kg}$ de Au recuperado, se pierden entre 5 y $10 \mathrm{~kg}$ de mercurio, de los cuales según Sánchez y Cañón (2010), aproximadamente 50\% del mercurio presente en la descarga se dispone en el suelo y en el agua y $25 \%$ se emite a la atmósfera, lo que evita que $100 \%$ del mercurio presente en las descargas llegue en su totalidad a las fuentes hídricas. Por tanto, Mancera-Rodríguez et al. (2006), manifiestan que la contaminación por mercurio y otros metales pesados es muy difícil de detectar a través

Tabla 4

Criterios de calidad del agua para consumo humano y uso doméstico, según el Decreto 1594 de 1984 y otras normativas internacionales

\begin{tabular}{|c|c|c|c|c|c|}
\hline \multirow[t]{2}{*}{ Parámetro } & \multicolumn{2}{|c|}{ Decreto 1594 de 1984} & Canadá & OMS & Panamá \\
\hline & $\begin{array}{l}\text { Criterios de calidad admisibles } \\
\text { para la destinación del recurso } \\
\text { para consumo humano y } \\
\text { doméstico que para su po- } \\
\text { tabilización se requiere solo } \\
\text { desinfección }\end{array}$ & $\begin{array}{l}\text { Criterios de calidad admisibles } \\
\text { para la destinación del recurso } \\
\text { humano y doméstico son los } \\
\text { que se relacionan a conti- } \\
\text { nuación, e indican que para } \\
\text { su potabilización se requiere } \\
\text { solamente tratamiento conven- } \\
\text { cional }\end{array}$ & Aguas des & das al c & sumo humano \\
\hline
\end{tabular}

\begin{tabular}{|c|c|c|c|c|c|}
\hline $\begin{array}{l}\text { Películas de grasas y } \\
\text { aceites (mg/l) }\end{array}$ & Ausentes & Ausentes & & & \\
\hline $\mathrm{pH}$ & $6.5-8,5$ & $5-9$ & $6,5-8.5$ & & \\
\hline Turbiedad (UJT) & 10 & 5 & $1-5$ & 5 & 10 \\
\hline Mercurio (mg/l) & 0,002 & 0,002 & & & \\
\hline
\end{tabular}




\section{Bioetnia Volumen 10, 2013}

del monitoreo medioambiental, debido a que las técnicas de análisis son específicas y costosas, adicionalmente, las concentraciones en el medio suelen ser más bajas que las encontradas en los sedimentos, o en las especies de fauna y flora presentes en los cuerpos de agua, por esto en ocasiones, una baja concentración en la columna de agua no necesariamente indica baja contaminación o poca utilización del elemento.

En zonas de alta presión minera a nivel nacional $\mathrm{y}$ regional, y en especial en el Distrito Minero del San Juan, el mercurio se ha constituido en uno de los elementos de contaminación más importantes con efectos sobre la salud pública. Lebel et al. (1996), han establecido que las personas o poblaciones expuestas a niveles bajos pueden desarrollar alteraciones en las funciones del sistema nervioso, el cual es esencialmente sensible al metil-mercurio con consecuencias neurofisiológicas, sobre todo en el desarrollo de los fetos y en los niños. Por otro lado, Brack et al. (2011), manifiestan que el mercurio en el agua es muy peligroso debido al proceso de bioacumulación (incremento de la concentración de mercurio en un organismo vivo a través del tiempo) y la biomagnificación (capacidad del metal de presentarse en bajas concentraciones en organismos al principio de la cadena trófica y en mayor proporción a medida que se asciende), razón por la cual los animales predadores son los que más mercurio tienen en sus tejidos y cuyo consumo implica más riesgo para las personas.

En cuanto a los sólidos totales y sólidos suspendidos, se registraron concentraciones que oscilaron entre $12 \mathrm{y}$ $120 \mathrm{mgl}^{-1} \mathrm{y}$ entre $7 \mathrm{y} 42 \mathrm{mgl}^{-1}$ antes de los vertimientos, y entre 410 y $10.230 \mathrm{mgl}^{-1} \mathrm{y}$ entre 356 y $8.799 \mathrm{mgl}^{-1}$ durante y después de los vertimientos respectivamente. Estas concentraciones de ST y SS, tuvieron una influencia directamente proporcional con la turbiedad, pues el comportamiento de esta variable estuvo influidoo por los sólidos (Tabla 1). Los altos niveles de sólidos, limitan el desarrollo de los procesos biológicos y la presencia de especies ícticas, por lo que, Brack et al. (2011) manifiestan que la presencia de ellos genera modificaciones en el hábitat de los organismos acuáticos, en sus hábitos migratorios y en el desarrollo de los huevos de peces. Las concentraciones de estos dos parámetros variaron tanto entre las estaciones de muestreos que el valor p estuvo por debajo de 0,005. Así, para los sólidos suspendidos el valor $\mathrm{p}$ fue 0,0244399 , y para los sólidos totales de 0,0249144; lo que indica que se presentó una diferencia estadísticamente significativa entre las medianas de estas variables por estaciones de muestreos con un nivel de $95 \%$ de confianza. Entre las estaciones de muestreo se resalta la diferencia estadística significativa que presentaron tanto los sólidos totales, como los sólidos suspendidos entre las estaciones antes y durante (SS valor p 0,000 , ST valor p de 0,0001 ) y entre las estaciones antes y después (valor p para ambas variables de 0,0000) (Tabla 2).

Aunque los SS no se encuentran referenciados en la normatividad colombiana, Ramírez y Viña(1998), consideran que aguas con valores mayores a $150 \mathrm{mgl}^{-1}$ indican fuerte contaminación, porque podría dificultar la penetración de la luz y el desarrollo de procesos químicos en presencia de oxígeno requeridos por organismos acuáticos específicos. Por su parte, la Fundación Mapfre (2005), indica que los sólidos en suspensión absorben la radiación solar y disminuyen la actividad fotosintética de la vegetación acuática. Al mismo tiempo obstruyen los cauces, intervienen en los procesos de producción industrial, pueden corroer los materiales y encarecer el costo de depuración del agua. De ahí que las altas concentraciones de estos elementos no solo implican impactos a nivel biológico y ecosistémico sino que también generan dificultades a nivel socioeconómico. En consecuencia se considera que los vertimientos mineros aumentan las concentraciones tanto de sólidos totales como de sólidos suspendidos del agua de las fuentes hídricas intervenidas.

En conclusión, se considera que los vertimientos mineros si influyen estadísticamente y de manera significativa sobre las características fisicoquímicas del agua de las fuentes hídricas receptoras, sobre todo entre las medianas de los parámetros como sólidos totales, sólidos suspendidos, turbiedad, y DQO, aunque para estos dos últimos solo se presentaron diferencias estadísticas significativas entre las estaciones antes y durante, y entre antes y después de los vertimientos mineros. Por otro lado, aun cuando no se hayan evidenciado diferencias estadística significativas entre las medianas de las concentraciones de mercurio y grasas y aceites, para algunos casos se encontraron por fuera de los valores establecidos por la normatividad nacional e internacional en aguas para consumo humano y uso doméstico y en aguas para conservación de flora y fauna acuática, a diferencia del $\mathrm{pH}$, que si bien presentó variaciones, estuvo en el rango establecido para ambos usos. En cuanto a la $\mathrm{BBO}$, esta fue constante y relativamente muy baja para todos los puntos analizados en las cuatro fuentes hídricas.

Finalmente, al comparar los resultados de las variables en cada una de las estaciones de muestreos, se observa que los mejores resultados se registraron en las estaciones antes de los vertimientos mineros, especialmente en los ríos Pureto y Tadocito, porque para la quebrada Tapacundó hubo reportes de altos niveles de turbiedad y de sólidos suspendidos. Para el caso de las estaciones de muestreos ubicadas durante y después de los vertimientos, las características fisicoquímicas del agua en la totalidad de los parámetros analizados se encontraban alteradas, gracias a la influencia que ejerce la carga contaminante presente en los efluentes mineros, lo que podría limitar el uso del agua para el desarrollo de diferentes actividades antrópicas, en 


\section{Vertimientos mineros y fuentes hídricas. FM Medina}

especial para consumo humano y uso doméstico y para conservación de flora y fauna acuática.

\section{Consideraciones finales}

Los efectos de los efluentes mineros generados sobre las características fisicoquímicas del agua de las fuentes hídricas superficiales, son el resultado de la inadecuada utilización de sustancias tóxicas, la falta de implementación de tecnologías limpias durante el proceso, conciencia ambiental de los mineros, control ambiental por parte del Estado (instituciones ambientales y minera) e implementación de medidas de manejo ambiental para la utilización del recurso agua.

En la minería se alcanzan descargas, con altos contenidos de contaminantes como sedimentos, grasas y aceites, y sustancias químicas como mercurio, los cuales al diluirse producen alteraciones en la calidad del agua, afectando sus condiciones naturales, limitando el uso del recurso, dificultando el mantenimiento de especies acuáticas y aumentando el riesgo de contaminación mercurial para las personas de las comunidades rivereñas principalmente. Y como consecuencia de esta problemática las poblaciones aledañas a las fuentes hídricas superficiales intervenidas, se están viendo altamente afectadas, pues, el transporte de altos contenidos de sedimentos y otros contaminantes, sumado a la remoción del suelo y la destrucción de la vegetación rivereña, ocasionan no solo la sedimentación de los cuerpos de aguas y con ello la reducción del recurso ictiológico y la vida acuática en general, sino que también aumentan los riesgos de deslizamientos, inundaciones y afectan la navegabilidad.

Lo anterior hace necesaria y urgente la implementación de medidas de manejo durante los procesos de extracción de metales a cielo abierto, que contribuyan a la reducción de las cargas contaminantes generadas en el lavado de la grava, e impedir su llegada de manera directa a los cuerpos de aguas.

De igual manera, se deben implementar monitoreos periódicos de las descargas antes de su vertimiento, garantizando de esta manera que el agua, cumplan con los criterios de calidad para vertimientos establecidos por el Decreto 1594 de 1984.

\section{Literatura citada}

Barrenechea Martel A. 2013. Aspectos fisicoquimicos de la calidad del agua. [fecha de consulta 2 de octubre del 2014]. Disponible en: http://cdam. minam.gob.pe:8080/bitstream/123456789/109/2/CDAM0000012-2. pdf pp 5-47.

Brack A, Ipenza C, Álvarez J, Sotero. 2011. Minería aurifera en Madre de Dios y contaminación con mercurio. Una bomba de tiempo. Lima: Ministerio de Ambiente; pp. 30, 56.

Convenio de Donación IDRC-IIAP, Proyecto $\mathrm{N}^{\circ} 050317-045$ del 19 de octubre de 2004. Diagnóstico situacional de la minería artesanal y en pequeña escala desarrollada por afrocolombianos en territorios colectivos de comunidades negras en el Chocó Biogeográfico. Quibdó: IDRC-IIAP; pp 17. Corporación Autónoma para el Desarrollo Sostenible del Chocó (CODECHOCO). 2010. Situación de la minería en el departamento del Chocó. Quibdó: CODECHOCO; 4 pp.

Defensoría del Pueblo. 2010. La minería de hecho en Colombia. Bogotá: Defensoría del Pueblo; pp 11.

Fundación Mapfre. 2005. Manual de contaminación ambiental. Itsemap ambiental. Parte I. [fecha de consulta 14 de julio de 2012]. Disponible en: http://acontaminacionambientallerp2012.blogspot.com/2012/06/ efectos-de-la-contaminacion-de-las.html

Instituto Catalán de Tecnología. 2004. Medio ambiente industrial. Barcelona: ICT.

Lebel J, Roulet M, Mergler D, Lucotte M, Larribe F. 1996. Fish diet and mercury expose in a riparian amazonian population. Water, air and soil pollution, 97: 31-44.

Mancera N, Rodríguez R. 2006. Estado del conocimiento de las concentraciones de mercurio y otros metales pesados en peces dulceacuícolas de Colombia. Acta Biol Colomb. 11 (1): 3-23.

Ministerio de Ambiente. Guías de Calidad para Agua de Bebida del Canadá [fecha de consulta 7 de octubre de 2014]. Disponible en: http://cdam. minam.gob.pe/publielectro/calidad\%20ambiental/normasrecursoagua. pdf

Organización Mundial de la Salud (OMS). Guias de calidad para aguas de consumo humano. $3^{\mathrm{a}}$ ed. Ginebra: OMS.

Ramírez A, Viña G. 1998. Limnología colombiana. Aportes a su conocimiento y estadísticas de análisis. Citado por Instituto de Investigaciones Ambientales del Pacífico. Chocó Biogeográfico 2010. Evaluación de las características ambientales y socioeconómicas del recurso hídrico en los municipios de Tadó, Istmina, Condoto (Chocó), Iscuandé y El Charco (Nariño). Quibdó: IIAP.

República de Colombia. 1984. Decreto 1594 de 1984. Usos del agua y residuos líquidos. Diario Oficial 36700 de julio 26 de 1984.120 pp.

Sánchez D, Cañón J. 2010. Análisis documental del efecto de vertimientos domésticos y mineros en la calidad del agua del río Condoto (Chocó, Colombia). Rev Gestión y Ambiente. 13 (3): 126.

Vargas L. 2012. Análisis de los impactos generados por la minería de oro y platino a cielo abierto sobre los recursos hídricos a partir de la cuantificación del consumo de agua y la carga contaminante de los vertimientos. Rev Bioetnia. 9 (2): 203-14. 